\title{
Analgesic effects of maternal breast milk and sucrose in early, full, and late-term neonates
}

\author{
Brenda B. Pérez-Méndez ${ }^{1}$, Erika del C. Ochoa-Correa ${ }^{1 *}$, Consuelo Treviño-Garza ${ }^{1}$, \\ Dolores C. Cortés-Flores², Jaime I. Bocanegra-Cedillo ${ }^{1}$, Anahí Flores-Rodríguez ${ }^{1}$, \\ Isaías Rodríguez-Balderrama ${ }^{1}$, and Manuel E. de la O-Cavazos ${ }^{1}$ \\ ${ }^{1}$ Pediatrics Department, University Hospital, Autonomous University of Nuevo Leon, Monterrey, Nuevo Leon; ${ }^{2}$ Oncology Department, Hospital Infantil \\ de México Federico Gómez, Mexico City, Mexico
}

\begin{abstract}
Objective: The objective of the study was to compare the analgesic effect of sucrose and breastfeeding newborns during heel lancing at the "Dr. José Eleuterio González" University Hospital. Materials and methods: A prospective randomized study was performed, in which pain intensity was compared through the premature infant pain profile (PIPP) scale during heel lancing as part of routine medical care screening. Newborns were divided into two groups: sucrose and breastfed. In addition, the population of each group was subdivided into early-term infants (37.0-38.6 weeks of gestation [WOG]), full-term infants (39.0-39.6 WOG), and late-term infants (40.0-41.6 WOG). Results: It was found that before a pain stimulus, the breastfeeding group had a more significant percentage of newborns who were asleeplactive with eyes closed and facial movements $(p=0.001)$. After the heel lancing, the pain intensity evaluated by the PIPP was moderated for both groups, and no difference was found. When comparing each group according to gestational age, there was no significant difference in pain intensity. Conclusion: Breastfeeding and sucrose are equally effective as non-pharmacological analgesics during metabolic screening in early-, full-, and late-term neonates.
\end{abstract}

Key words: Breastfeeding. Sucrose. Analgesia. Newborn.

\section{Introduction}

Pain is an unpleasant emotional and sensory experience linked to actual or potential tissue damage ${ }^{1}$. During the care of newborns in a hospital setting, there are certain procedures performed in both sick and healthy neonates, which are considered to be painful ${ }^{2,3}$. The prevention of pain in newborns is expected from parents ${ }^{4}$. Painful stimuli during early stages of development can result in changes in the way pain are perceived in adult life, and infants are more susceptible to these effects in the long term ${ }^{5}$.
Since neonates cannot verbally express pain, the use of a scale to objectively measure the intensity of pain is necessary. The premature infant pain profile (PIPP) is valid for preterm and term neonates ${ }^{6,7}$.

For years, we have searched for alternatives to pharmacological methods used for pain in minor procedures on newborns. Among the non-pharmacological alternatives for pain management in neonates are pacifiers (suckers), sucrose solutions, breastfeeding, olfactory stimuli, and the Kangaroo mother care technique ${ }^{3,8-10}$. Analgesic effects of sucrose are mediated by the activation of endogenous opioid pathways, which produce

\section{Correspondence:}

*Erika del C. Ochoa-Correa

E-mail: erika8ac@ hotmail.com
Available online: 09-12-2019

Date of reception: 28-06-2019

Date of acceptance: 28-10-2019 DOI: 10.24875/RMU.19000103
Medicina Universitaria. 2019;21(4):169-174 www.medicinauniversitaria.org

Autónoma de Nuevo León. Published by Permanyer. This is an open access article under the CC BY-NC-ND license (http://creativecommons.org/licenses/by-nc-nd/4.0/). 
its release in response to sweet flavors ${ }^{11}$. The most significant effect of the latter begins approximately 2 min after administration and lasts for approximately $4 \mathrm{~min}^{12}$. The effectiveness of sucrose to treat pain in neonates decreases with postnatal age and is not useful in the first $24 \mathrm{~h}$ of postnatal life $e^{12,13}$. This could be a result of the fact that during the $1^{\text {st }} \mathrm{h}$ after birth, there are significant concentrations of endogenous opioids; therefore, sucrose does not offer any additional effects to the physiological levels during postnatal adaptation.

Breastfeeding has an analgesic effect given by the presence of the mother, providing peace, skin on skin contact, a sweet taste, and a distraction to the infant ${ }^{14,15}$. Moreover, it contains a higher concentration of tryptophan compared to feeding formulas, which is a melatonin precursor that has proven to increase $\beta$-endorphin concentration ${ }^{16}$.

Routinely, pain control methods for minor procedures, such as heel lancing for sample taking in neonate screenings, are not usually performed at the newborn nursery. Since painful stimuli during childhood result in changes in the way pain are perceived in adult life, it is important to find an effective analgesic procedure, and one that is minimally invasive, for pain control of full-term neonates, especially for minor procedures such as neonatal screenings, and proves if perception of pain is different in early-term (37.0 - 38.6 weeks of gestation [WOG]), full-term (39.0 - 40.6 WOG), and lateterm (41.0 - 41.6 WOG) newborns ${ }^{17}$.

\section{Materials and methods}

A controlled, prospective, and randomized clinical assay were conducted to compare the analgesic effect of breastfeeding and sucrose during heel lancing, approved by the Ethics Committee of the School of Medicine at the Autonomous University of Nuevo Leon.

The study was performed between April 2015 and September 2015 in the rooming-in (mother and child together 24 h) at the "Dr. Jose Eleuterio Gonzalez" University Hospital.

The inclusion criteria included term newborns (37-41 WOG) that were born at the delivery area at the aforementioned institution, who were accommodated with the mother, healthy, with an age of 48-72 $\mathrm{h}$ of life, an Apgar score of $\geq 7$ at $5 \mathrm{~min}$ of life, and with a fasting period of at least $30 \mathrm{~min}$.

The study excluded patients who were a product of a high-risk pregnancy, those who presented medical instability, use of general anesthesia in the mother at the moment of birth, maternal use of opioids, and administration of naloxone or phenobarbital to the mother in the $48 \mathrm{~h}$ previous to birth, or those who were not breastfed. In addition, patients who had incomplete data were excluded, as well.

The painful procedure studied was heel lancing as part of routine medical care screening, performed with an automated lancet $1 \mathrm{~mm}$ deep with a $2.5 \mathrm{~mm}$ incision (Quikheel ${ }^{\mathrm{TM}}$ ). A single nurse, who was trained to take samples for neonatal screening, performed the heel punctures. Sampling was conducted at $72 \mathrm{~h}$ of life.

In the sucrose group, 2 min after the screening puncture, $1 \mathrm{ml}$ of sucrose solution was administered at $25 \%$ through a drip, while in the breastfed group, the newborns were left in contact with their mother and breastfed during the entire procedure. Subsequently, a general medical evaluation was performed; both heart rate and oxygen saturation were measured, leaving the newborn monitored during the whole procedure through pulse oximetry. The procedures were recorded and later reviewed to revise the research parameters. Afterward, the videos were examined by a neonatologist for the evaluation of pain.

The PIPP was used for the evaluation of pain, consisting of 7 items, which include gestational age, behavioral state, physiological parameters (changes in heart rate and oxygen saturation), and facial gestures (frown, squinting, and nasolabial groove). These items are added up, giving a score that divides pain into mild, moderate, and severe categories ${ }^{6}$. This score was utilized since it is routinely used in the neonatology area at the institution, it is validated for its use in premature and term neonates, and it also has a section for newborns older than 36 WOG.

The sample size was 63 newborns in each group to reach $80 \%$ potency to detect a difference of 2 points in the PIPP scale (assuming an SD of 4) between breastfeeding and sucrose, with a significance level of 0.05 (two tailed). The randomization design was generated using the website randomization.com /http://www.randomization.com〉.

Descriptive statistics were utilized, including frequencies and percentages for categorical variables, means, medians, standard deviations, and ranges for quantitative variables according to their distribution. Comparisons between groups were performed using parametric and non-parametric tests for quantitative variables and Chi-square or Fisher's exact statistic for categorical variables. An alpha value of 0.05 was considered statistically significant. We used the statistical program SPSS 20 (SPSS, Chicago, US). 
Table 1. Demographic characteristics of the population

\begin{tabular}{|l|c|c|c|}
\hline Characteristics & Breastfeeding $(\mathbf{n}=\mathbf{7 3})$ & Sucrose (n= 89) & $\mathbf{p}$ \\
\hline Gestational age, median (RIO) & $38.6(38.0-39.5)$ & $39.4(38.4-40.1)$ & $0.0007^{1}$ \\
\hline Weight at birth (g), average (SD) & $3187(425)$ & $3211(564)$ & $0.76^{2}$ \\
\hline Length at birth (cm), average (SD) & $49.9(1.8)$ & $49.4(5.2)$ & $0.48^{2}$ \\
\hline Apgar at 5 min, $\mathrm{n}(\%)$ & $1(1.4)$ & $2(2.2)$ & $0.38^{4}$ \\
\hline 8 & $70(95.9)$ & $80(89.9)$ & $7(7.9)$ \\
\hline 10 & $2(2.7)$ & $39(43.8)$ & $0.22^{3}$ \\
\hline
\end{tabular}

1: Mann-Whitney U-test; ${ }^{2}$ : Student's t-test; ${ }^{3}$ : Pearson's Chi-square; ${ }^{4:}$ Fisher's exact statistic. SD: standard deviation.

Table 2. Heart rate and oxygen saturation

\begin{tabular}{|c|c|c|c|}
\hline Parameter of the PIPP scale & Breastfeeding & Sucrose & Student's t-test (p) \\
\hline HR before painful stimulus, average (SD) & $132 \pm 17$ & $130 \pm 17$ & $0.39^{1}$ \\
\hline HR after painful stimulus, average (SD) & $141 \pm 20$ & $140 \pm 19$ & $0.58^{1}$ \\
\hline Oxygen saturation before painful stimulus, average (SD) & $96 \pm 2.6$ & $96 \pm 3.0$ & $0.77^{1}$ \\
\hline Oxygen saturation after painful stimulus, average (SD) & $95 \pm 4.2$ & $96 \pm 3.2$ & $0.09^{1}$ \\
\hline
\end{tabular}

HR: heart rate; PIP: premature infant pain profile; SD: standar deviation.

\section{Results}

A total of 180 patients were included, of which 17 were excluded due to incomplete data and and one because a complete atrioventricular block was found. Of the remaining 162 patients, 73 were placed in the breastfeeding group and 89 in the sucrose group.

Mean gestational age in weeks was 39 (interquartile range [IQR] 38.1-40), 84 patients were female (51.9\%), mean weight was $3200.9 \mathrm{~g} \mathrm{(} \pm 505.4)$, and mean height was $49.6 \mathrm{~cm}( \pm 4.06)$.

When comparing demographic characteristics of the population, a difference was found in gestational age with 39.4 (IQR 38.4 - 40.1) for the sucrose group and 38.6 (IQR 38.0 - 39.5) for the breastfeeding group $(p=0.039)$. For the rest of the variables, no difference was found between both groups (Table 1).

Heart rate and oxygen saturation were measured previous and after the painful stimulus within the quantitative variables of the PIPP scale, without finding differences in any of the measurements when we compared the breastfeeding group and the sucrose group (Table 2).

Among the qualitative variables if the PIPP scale, a difference in the behavioral state was found previous to the painful stimulus, finding that 53 patients $(72.6 \%)$ had their eyes closed and presented facial movements (score of 2) in the breastfeeding group and 54 patients $(60.7 \%)$ were awake with their eyes open and facial movements (score 0$)$ in the sucrose group $(p<0.001)$. No statistically significant differences were found in the rest of the qualitative variables (Table 3).

There were no differences in the total score of the scale, being 11 (IQR 7 - 12) in the breastfeeding group and 11 (IQR $8-13)$ in the sucrose group $(p=0.45)$. No differences were found in pain level after the stimulus in both groups $(p=0.09)$.

When dividing the groups according to term classification, there were no differences in the manifestation of pain in early-term, full-term, and/or late-term neonates (Table 4).

\section{Discussion}

In the analysis of demographic data, a statistically significant difference was found concerning gestational age. Differences in the expression of pain between preterm and term neonates are widely reported ${ }^{18,19}$. The difference in gestational age has been proven to have effects on the clinical outcome of term neonates; 
Table 3. Behavior and facial gestures

\begin{tabular}{|c|c|c|c|c|c|c|c|}
\hline \multirow{2}{*}{$\begin{array}{l}\text { Parameter of the PIPP } \\
\text { scale }\end{array}$} & \multirow[t]{2}{*}{ Points } & \multicolumn{3}{|c|}{ Before } & \multicolumn{3}{|c|}{ After } \\
\hline & & Breastfeeding & Sucrose & $\begin{array}{c}\text { Pearson's } \\
\text { Chi-square (p) }\end{array}$ & Breastfeeding & Sucrose & $\begin{array}{l}\text { Fisher's exact } \\
\text { statistic }(p)\end{array}$ \\
\hline \multirow[t]{4}{*}{ Behavior, n (\%) } & 0 & 17 (23.3) & $54(60.7)$ & $<0.001^{2}$ & $9(12.3)$ & 17 (19.1) & $0.588^{2}$ \\
\hline & 1 & $1(1.4)$ & $3(3.4)$ & & 0 & $1(1.1)$ & \\
\hline & 2 & $53(72.6)$ & $32(36)$ & & $63(86.3)$ & $70(78.7)$ & \\
\hline & 3 & $2(2.7)$ & 0 & & $1(1.4)$ & $1(1.1)$ & \\
\hline \multirow[t]{4}{*}{ Puckered eyebrows, n (\%) } & 0 & $65(89)$ & $80(89.9)$ & $0.95^{2}$ & $12(16.4)$ & $8(9)$ & $0.435^{1}$ \\
\hline & 1 & $5(6.8)$ & $5(5.6)$ & & $11(15.1)$ & $11(12.4)$ & \\
\hline & 2 & $2(2.7)$ & $3(3.4)$ & & $13(17.8)$ & $16(18)$ & \\
\hline & 3 & $1(1.4)$ & $1(1.1)$ & & $37(50.7)$ & $54(60.7)$ & \\
\hline \multirow[t]{4}{*}{ Eyes shut, $\mathrm{n}(\%)$} & 0 & $67(91.8)$ & $84(94.4)$ & $0.78^{2}$ & $9(12.3)$ & $9(10.1)$ & $0.16^{1}$ \\
\hline & 1 & $4(5.5)$ & $2(2.2)$ & & $15(20.5)$ & $8(9)$ & \\
\hline & 2 & $1(1.4)$ & $2(2.2)$ & & $15(20.5)$ & $20(22.5)$ & \\
\hline & 3 & $1(1.4)$ & $1(1.1)$ & & $34(46.6)$ & $52(58.4)$ & \\
\hline \multirow[t]{4}{*}{ Nasolabial groove, $\mathrm{n}(\%)$} & 0 & $61(83.6)$ & $74(83.1)$ & $0.34^{2}$ & $9(12.3)$ & $3(3.4)$ & $0.07^{1}$ \\
\hline & 1 & $8(11)$ & $9(10.1)$ & & $13(17.8)$ & 10 (11.2) & \\
\hline & 2 & $2(2.7)$ & $6(6.7)$ & & $8(11)$ & 14 (15.7) & \\
\hline & 3 & $2(2.7)$ & 0 & & 43 (58.9) & $62(69.7)$ & \\
\hline
\end{tabular}

PIP: premature infant pain profile.

hence, a classification was made at the time of pregnancy resolution into three categories: early term (37 - 38.6 weeks), full term (39 - 40.6 weeks), and late term (41 - 41.6 weeks). We were unable to find literature reporting a difference in the expression of pain in term neonates according to the subclassification of the term neonate.

The PIPP score is applied in a simple manner and without the need for sophisticated equipment, in addition to the fact that it has been validated for its use in term neonates ${ }^{6,7}$. It has been shown that physiological parameters can be altered with other stimuli besides pain, such as hypervolemia, temperature, and mechanical ventilation, to name a few. Since our study population is healthy neonates, we considered that changes in these parameters are mainly linked to painful stimuli. Another benefit of the use of this score is that in addition to including physiological parameters, it includes the evaluation of behavioral state and facial gesticulation, which are other parameters linked to the expression of pain in this population ${ }^{20-22}$.

A puncture with an automatic lance causes less damage than one performed with a manual lance,
Table 4. Pain by term classification

\begin{tabular}{|c|c|c|c|}
\hline Pain intensity & Breastfeeding & Sucrose & $\mathbf{p}$ \\
\hline $\begin{array}{l}\text { Early term, } \mathrm{n}(\%) 69 \\
\text { Light }(<6 \text { points) } \\
\text { Moderate (6-12 points) } \\
\text { Severe (> } 12 \text { points) }\end{array}$ & $\begin{array}{c}8(11.6) \\
25(36.2) \\
4(5.8)\end{array}$ & $\begin{array}{c}6(8.7) \\
17(24.7) \\
9(13)\end{array}$ & $0.18^{1}$ \\
\hline $\begin{array}{l}\text { Full term, } \mathrm{n}(\%) 83 \\
\text { Light }(<6 \text { points) } \\
\text { Moderate (6-12 points) } \\
\text { Severe (> } 12 \text { points) }\end{array}$ & $\begin{aligned} 8 & (9.6) \\
18 & (21.7) \\
7 & (8.4)\end{aligned}$ & $\begin{array}{c}6(7.3) \\
31(37.3) \\
13(15.7)\end{array}$ & $0.34^{1}$ \\
\hline $\begin{array}{l}\text { Late term, } \mathrm{n}(\%) 10 \\
\text { Light }(<6 \text { points) } \\
\text { Moderate (6-12 points) } \\
\text { Severe }(>12 \text { points })\end{array}$ & $\begin{array}{c}1(10) \\
2(20) \\
0\end{array}$ & $\begin{array}{l}1(10) \\
3(30) \\
3(30)\end{array}$ & $0.38^{1}$ \\
\hline Early term, median (RI0) & $9.3(6-12)$ & $11(7-13)$ & $0.42^{2}$ \\
\hline Full term, median (RIO) & $10.3(9.5-12.5)$ & $11(9-13)$ & $0.71^{2}$ \\
\hline Late term, median (RIO) & $11(3-11)$ & $12(8-14)$ & $0.38^{2}$ \\
\hline \multicolumn{4}{|c|}{$\begin{array}{l}\text { 1: Pearson's Chi-square; }{ }^{2} \text { : Mann-Whitney U-test. } \\
\text { besides being a standardized stimulus }{ }^{23} \text {. Moreover, } \\
\text { by having this procedure always performed by the } \\
\text { same personnel, they were able to guarantee that the } \\
\text { same heel lancing technique was used, hence, }\end{array}$} \\
\hline
\end{tabular}


making it improbable for the technique to influence the results.

Regarding the use of the mother's breast for pain control, there is a systemic revision which found it to reduce the increase in heart rate, the amount of crying time, the duration of the first cry, and the total amount of crying, compared with containment measures. The PIPP scale and other scales were less in the breastfeeding group compared to containment measures ${ }^{24}$.

The analgesic effect of sucrose has also been evaluated in a systemic revision, where it was assessed with different stimuli, including heel lancing. In this revision, a decrease in the PIPP scale was found after a puncture when compared with a placebo ${ }^{25}$. However, in this study, we compared the effect of sucrose with another non-pharmacological alternative to treat pain, i.e., breastfeeding; the reason for not adding a placebo group is because it has been widely proven that most times, the heel lancing causes moderate-to-severe pain in neonates and adding it was not considered prudent.

This study did not find differences in the levels of pain or PIPP score when comparing the use of breastfeeding or sucrose in pain control subsequent to heel lancing. These findings concur with those reported in literature, where the difference in the PIPP scale was not statistically significant when comparing breastfeeding to sucrose ${ }^{24}$.

Within the limitations of the study, there is the fact that within the breastfeeding group, the newborns were kept skin on skin with the mother, which has also been reported to have an analgesic effect ${ }^{10}$.

Another limitation is that the patients in the breastfeeding group had a gestational age lower than the patients in the sucrose group. However, the results coincide with those reported in literature. Therefore, we consider that it is not likely that the difference in gestational age in both groups represents a bias. When we subclassify the neonates as early term, full term, and late term, the sample by classification becomes small. Thus, statistical potency may have been lost, resulting in the need for further studies to prove our findings.

Since there are no differences in pain management using sucrose or breastfeeding, we suggest the use of the latter because it requires no material for its application and does not involve more costs for its use in pain control during minor procedures routinely performed in a healthy newborn's care.

\section{Conclusion}

Breastfeeding and sucrose are equally as effective as non-pharmaceutical analgesics during the performance of a metabolic screening, that is, term neonates.

\section{Conflicts of interest}

Authors declare that they have no conflicts of interest.

\section{Ethical disclosures}

Protection of human and animal subjects. The authors declare that the procedures followed were in accordance with the regulations of the relevant clinical research ethics committee and with those of the Code of Ethics of the World Medical Association (Declaration of Helsinki).

Confidentiality of data. The authors declare that they have followed the protocols of their work center on the publication of patient data.

Right to privacy and informed consent. The authors have obtained the written informed consent of the patients or subjects mentioned in the article. The corresponding author is in possession of this document.

\section{References}

1. International Association for the Study of Pain (IASP) Terminology; 2017. Available from: https://www.iasp-pain.org/Education/Content.aspx?ItemNumber=1698. [Last accessed on 2019 Mar 26].

2. Anand KJ, International Evidence-Based Group for Neonatal Pain. Consensus statement for the prevention and management of pain in the newborn. Arch Pediatr Adolesc Med. 2001;155:173-80.

3. Allegaert K, van den Anker JN. Neonatal pain management: still in search for the holy grail. Int J Clin Pharmacol Ther. 2016;54:514-23.

4. Beier $\mathrm{KH}$, Heegaard W, Rusnak RA. Acute neonatal scalp abscess and E. coli bacteremia in the ED. Am J Emerg Med. 1999;17:241-3.

5. Fitzgerald M, Beggs S. The neurobiology of pain: developmental aspects. Neuroscientist. 2001;7:246-57.

6. Ballantyne M, Stevens B, McAllister M, Dionne K, Jack A. Validation of the premature infant pain profile in the clinical setting. Clin $\mathrm{J}$ Pain. 1999;15:297-303.

7. Slater R, Cantarella A, Franck L, Meek J, Fitzgerald M. How well do clinical pain assessment tools reflect pain in infants? PLoS Med. 2008;5:e129.

8. Pillai Riddell RR, Racine NM, Gennis HG, Turcotte K, Uman LS, Horton RE, et al. Non-pharmacological management of infant and young child procedural pain. Cochrane Database Syst Rev. 2015;10:CD006275.

9. De Clifford-Faugère G, Lavallée A, Aita M. Olfactive stimulation interventions for managing procedural pain in preterm and full-term neonates: a systematic review protocol. Syst Rev. 2017;6:203.

10. Johnston C, Campbell-Yeo M, Disher T, Benoit B, Fernandes A, Streiner D, et al. Skin-to-skin care for procedural pain in neonates. Cochrane Database Syst Rev. 2017;2:CD008435

11. Barr RG, Quek VS, Cousineau D, Oberlander TF, Brian JA, Young SN, et al. Effects of intra-oral sucrose on crying, mouthing and hand-mouth contact in newborn and six-week-old infants. Dev Med Child Neurol. 1994;36:608-18.

12. Taddio A, Shah V, Hancock R, Smith RW, Stephens D, Atenafu E, et al. Effectiveness of sucrose analgesia in newborns undergoing painful medical procedures. CMAJ. 2008:179:37-43.

13. Blass EM, Watt LB. Suckling and sucrose-induced analgesia in human newborns. Pain. 1999;83:611-23.

14. Blass EM. Milk-induced hypoalgesia in human newborns. Pediatrics. 1997:99:825-9. 
15. Barrett T, Kent S, Voudouris N. Does melatonin modulate beta-endorphin, corticosterone, and pain threshold? Life Sci. 2000;66:467-76.

16. Weissman A, Aranovitch M, Blazer S, Zimmer EZ. Heel-lancing in newborns: behavioral and spectral analysis assessment of pain control methods. Pediatrics. 2009;124:e921-6.

17. Spong CY. Defining "term" pregnancy: recommendations from the defining "Term" pregnancy workgroup. JAMA. 2013;309:2445-6.

18. Gibbins S, Stevens B, McGrath PJ, Yamada J, Beyene J, Breau L, et al. Comparison of pain responses in infants of different gestational ages. Neonatology. 2008;93:10-8.

19. Johnston CC, Stevens B, Craig KD, Grunau RV. Developmental changes in pain expression in premature, full-term, two and four-month-old infants. Pain. 1993;52:201-8.

20. Relland LM, Gehred A, Maitre NL. Behavioral and physiological signs for pain assessment in preterm and term neonates during a nociception-specific response: a systematic review. Pediatr Neurol. 2019;90:13-23.
21. Maxwell LG, Malavolta CP, Fraga MV. Assessment of pain in the neonate. Clin Perinatol. 2013;40:457-69.

22. Harrison D, Evans $C$, Johnston L, Loughnan P. Bedside assessment of heel lance pain in the hospitalized infant. J Obstet Gynecol Neonatal Nurs. 2002;31:551-7.

23. Vertanen $\mathrm{H}$, Fellman V, Brommels M, Viinikka L. An automatic incision device for obtaining blood samples from the heels of preterm infants causes less damage than a conventional manual lancet. Arch Dis Child Fetal Neonatal Ed. 2001;84:F53-5.

24. Shah PS, Herbozo C, Aliwalas LL, Shah VS. Breastfeeding or breast milk for procedural pain in neonates. Cochrane Database Syst Rev. 2012 12:CD004950.

25. Stevens B, Yamada J, Ohlsson A, Haliburton S, Shorkey A. Sucrose for analgesia in newborn infants undergoing painful procedures. Cochrane Database Syst Rev. 2016;7:CD001069. 